HERRERO, María Belén; CARBONETTI, Adrián. La mortalidad por tuberculosis en Argentina a lo largo del siglo XX. História, Ciências, Saúde - Manguinhos, Rio de Janeiro, v.20, n.2, abr.-jun. 2013, p.521-536.

Resumen

El objetivo de este artículo es realizar un análisis de la curva de mortalidad por tuberculosis en Argentina a lo largo del siglo XX, desde 1911 hasta 2007. A partir de los datos obtenidos de diversas fuentes de carácter oficial se marcan las etapas históricas de dicha mortalidad, generando dentro de éstas subperíodos, a fin de entender el comportamiento de la mortalidad por tuberculosis, dependiendo del momento histórico. De esta forma, se vincula el desarrollo de la enfermedad con los dispositivos que se generaron para combatirla. Se realiza un análisis del comportamiento de la mortalidad por regiones del país, partiendo del supuesto que el desarrollo de la misma fue diferente según condiciones económicas y sociales y desarrollo sanitario.

Palabras clave: Argentina; tuberculosis; mortalidad; siglo XX.

Abstract

The aim of this article is to provide an analysis of the mortality curve for tuberculosis in Argentina throughout the twentieth century, from 1911 to 2007. Using data from various official sources, the mortality rate is divided into historical phases and sub-periods, in order to show how the behavior of tuberculosis mortality depended on the historical moment. Thus, the progress of the disease is linked to the methods that were developed to combat it. The fluctuations in mortality are analyzed by regions of the country, on the assumption that mortality varied according to economic and social conditions and the development of healthcare.

Keywords: Argentina; mortality; tuberculosis; twentieth century. 
$\mathrm{L}$ a humanidad ha padecido la tuberculosis durante miles de años. Se estima que el Mycobacterium tuberculosis, el agente causal descubierto por Robert Koch, podría haber matado más personas que cualquier otro agente patógeno a lo largo de la historia de la humanidad (Ritacco, Leão, Palomino, 2007). Se calcula que en el siglo XIX la tuberculosis acabó con la vida de una cuarta parte de la población adulta de Europa (Médicos Sin Fronteras, 2006). Durante ese período las terapias en las casas de curación o en los sanatorios eran las principales medidas de abordaje de la tuberculosis con el objetivo de aislar a los pacientes, romper la cadena de transmisión de la enfermedad, y ofrecerles buen clima, reposo y dieta adecuada (Ritacco, Leão, Palomino, 2007).

Posteriormente, y debido a la mejora de las condiciones de vida y al descubrimiento de los antibióticos, la morbi-mortalidad por tuberculosis había descendido considerablemente (Armus, 2007; Ritacco, Leão, Palomino, 2007). Si bien esta enfermedad continuaba afectando a las poblaciones más vulnerables, constituyendo uno de los principales problemas de salud pública y una de las primeras causas de muerte en varios países de menores ingresos, hacia los años cincuenta la mortalidad descendería notablemente gracias a los tratamientos, al igual que la incidencia de la enfermedad, principalmente en los países industrializados (Farmer, 1999).

A mediados del siglo XX, se comprueba la eficacia de la estreptomicina para inhibir el crecimiento del bacilo. Posteriormente en la década del setenta, la aparición de la rifampicina da lugar a la antibiótico-terapia, identificada como la medida más efectiva para controlar la infección en el paciente y, así, revertir su transmisibilidad.

Debido a varios factores, entre ellos, un brote de tuberculosis multirresistente (TB-MDR), en Nueva York, entre 1991 y 1993, y la creciente evidencia de la relación entre tuberculosis y el VIH/Sida, esta situación empezó a cambiar, hasta que en el año 1993 la Organización Mundial de la Salud (OMS) la declaró emergencia global.

Aunque en la actualidad la tuberculosis es curable, en el mundo mueren a causa de la enfermedad seis mil personas por día, una cada quince segundos, y más de dos millones anualmente; es la enfermedad infecciosa que causa mayor cantidad de enfermos y muertes en adultos a nivel mundial (Argentina, 2006). La mortalidad por tuberculosis varía dramáticamente en el mundo: no sólo veintidós países tienen el 80\% de los casos nuevos, sino que el 98\% de las muertes por tuberculosis se producen en países en desarrollo (OPS, 2004).

Según un informe de la OMS, en la Región de las Américas, en el 2006, se produjeron alrededor de 41 mil muertes, siendo las zonas de Latinoamérica y el Caribe las que han concentrado las mayores cifras. En la Argentina, la tuberculosis es uno de los principales problemas de salud pública. Cada año se diagnostican más de once mil nuevos casos y mueren más de ochocientas personas a causa de la enfermedad (Iner, 2009) y, como sucede a nivel mundial, la distribución geográfica de la enfermedad en nuestro país tampoco es uniforme (Iner, 2009).

La tuberculosis ha sido, tal vez, una de las dolencias que más ha llamado la atención de los científicos sociales que han trabajado y trabajan en la historia de la salud y la enfermedad. Por sus connotaciones sociales, políticas, económicas y culturales, que llegan hasta la actualidad, la tuberculosis formó y forma parte de la preocupación médico-estatal debido a que aún hoy sigue siendo un problema sanitario, especialmente para los sectores más pobres de la sociedad. 
Son numerosos los trabajos que se han realizado en América Latina, especialmente con la renovación que se llevó a cabo a partir de los años setenta del siglo pasado, renovación que también llevó a numerosos historiadores a tomarla como objeto de sus investigaciones (Bertolli Filho, 2001; Pôrto, 2000; Armus, 2007; Carbonetti, 1998; 2011, Idrovo, 2004; Beldarraín Chaple, 1998).

En Argentina la historia de la tuberculosis ha sido analizada desde diferentes perspectivas (Recalde, 1997; Armus, 2007; Carbonetti, 2011; Blinn Reber, 1999). Sin embargo, la mayoría de estos trabajos estuvieron circunscritos al análisis de las connotaciones sociales, culturales, médicas y demográficas sólo en algunas provincias de la Argentina, especialmente Buenos Aires y Córdoba y para un período de tiempo acotado, entre fines del siglo XIX y mediados del siglo XX.

Por otra parte, si bien se han desarrollado trabajos donde se permite apreciar la curva de mortalidad por tuberculosis a lo largo del pasado siglo XX, como por ejemplo el realizado para el caso de Cuba (González Ochoa et al., 2003), aún no se conocen trabajos para el caso de la Argentina.

El objetivo de este estudio es realizar una descripción y análisis de la mortalidad por tuberculosis en la Argentina desde comienzos del siglo XX hasta la actualidad, poniendo énfasis en el desarrollo de la curva a lo largo de la centuria, y de entender los cambios que se produjeron a lo largo de este período en la mortalidad por esta enfermedad en las distintas regiones del país, a partir de la hipótesis de que en la Argentina se dio un panorama heterogéneo dependiendo de las condiciones de vida y sanitarias de cada uno de los distritos que la componen.

Para realizar el análisis partimos de la teoría de la transición epidemiológica y de la transición de la mortalidad, retomando los estudios de Thomas McKeown y sus posteriores revisiones. Haremos especial hincapié en la tesis de Simon Szreter (1997a) que pone énfasis en las cuestiones de carácter político para explicar la disminución de la mortalidad.

Las fuentes para el análisis se basan especialmente en trabajos estadísticos realizados por médicos del Departamento Nacional de Higiene, del Ministerio de Salud de la Nación y en los informes epidemiológicos del Instituto Nacional de Enfermedades Respiratorias Dr. Emilio Coni que dispone de información de la mortalidad por tuberculosis desde mediados del siglo XX hasta la actualidad. El problema fundamental a la hora del análisis de las fuentes es el de la veracidad de las mismas, en especial en el período que va entre principios y mediados del siglo XX, momento en el cual la descentralización del sistema de salud, la falta de instituciones que centralizaron la estadística y la falta de médicos que diagnosticaran la causa de muerte permite pensar en cierto grado de subregistro, en especial en las provincias del Noroeste y Noreste, como se podrá apreciar en el apartado del análisis de las regiones. Ahora bien, por su peso proporcional en términos del total de la población argentina se trataría de un número escaso de subregistros, ya que este fenómeno se daría en regiones rurales con escaso número de población (Araoz, 1916, p.11). No obstante, consideramos como esencial la tendencia que ha tenido la enfermedad a lo largo del siglo XX, analizar los períodos y subperíodos y realizar hipótesis acerca del desarrollo que tuvo la misma. En ese sentido se realizó una comparación de las tasas de mortalidad del total de Argentina con las curvas de mortalidad de algunas ciudades como Córdoba y Buenos Aires, donde se realizaron trabajos desde la misma perspectiva que 
permiten considerar, por lo menos en el momento donde existen dudas, la veracidad de los datos; si la tendencia que siguieron fue la misma. Ese ejercicio permitió apreciar que en términos generales las tendencias de la mortalidad por tuberculosis serían similares a las de las ciudades antes anotadas, por lo cual creemos que es posible realizar el estudio.

Se considerarán dos etapas definidas a partir de la aplicación de medicamentos efectivos para combatir la tuberculosis: de 1911 a 1946 y de 1947 a 2007.

\section{La mortalidad por tuberculosis en Argentina a lo largo del siglo XX}

Antes del descubrimiento y aplicación de los medicamentos antituberculosos, y según la descripción de la historia natural de la tuberculosis, se conocía que aproximadamente el $50 \%$ de los pacientes con esta enfermedad morirían por esta causa. No obstante, durante la primera mitad del siglo XX ya se observaba una declinación de la mortalidad debido a factores variados y complejos. El hecho que la mortalidad comenzara a caer antes de las campañas antituberculosas del siglo - al menos en algunos países - se debe, probablemente, a los cambios producidos en el equilibrio natural entre agente, huésped, ambiente, estilo de vida, condiciones de vida y servicios de salud que determinan la ocurrencia y distribución geográfica de la tuberculosis (González Ochoa et al., 2003).

Sin embargo, a pesar de las mejoras ambientales y algunos adelantos tecnológicos en la lucha antituberculosa, el descenso en la mortalidad se mantuvo en forma suave hasta 1946. Será unos años más tarde, con la introducción de la estreptomicina e isoniacida, que se produjo una caída abrupta de la mortalidad en casi todo el mundo. La introducción de la quimioterapia y su perfeccionamiento han permitido que la curación de la tuberculosis sea posible en más del 95\% de los casos (Iner, 1988). En consecuencia, mientras que en 1900 no había un país que registrara una mortalidad por tuberculosis menor que cien por cien mil habitantes, ya en 1980 los países industrializados reportaban tasas menor que cinco por cien mil (González Ochoa et al., 2003).

En este sentido, la mortalidad por tuberculosis en Argentina muestra una evolución similar a la de la mayoría de los países occidentales en el siglo XX, con fluctuantes períodos de aumento y descenso, pero con una disminución constante a partir de 1918 que se hace más marcada a partir de 1947. El proceso, a partir de esta fecha, es probable que esté asociado a la introducción de la quimioterapia de la mano de la estreptomicina en primer lugar, y posteriormente con la isoniacida. El comportamiento que tuvo la mortalidad por tuberculosis a lo largo del siglo XX se puede apreciar en el Gráfico 1.

Desde 1911, período en que comienza nuestro estudio, hasta el año 2007, la mortalidad por tuberculosis cayó 66 veces, pasando de 134 a 2,02 muertes por cien mil habitantes apenas empezado el siglo XXI.

La caída de la mortalidad por tuberculosis tiene varias particularidades. Por un lado está inmersa en un proceso de reducción de la mortalidad general que se lo conoce como la transición de la mortalidad. Por el otro, en un cambio en las estructuras de causas de muertes que pasan de ser exógenas, provocadas por microrganismos, fundamentalmente, a causas de carácter endógenas dentro de las cuales predominan los accidentes cerebro vasculares, cardiovasculares y tumores provocadas por las condiciones de vida, estilos de 
vida y ambientales. Este proceso de cambio en la estructura de causas de muerte es conocido como transición epidemiológica (Carbonetti, Celton, 2007).

Por otra parte, esa reducción de la que hablábamos en el párrafo anterior no fue uniforme, sino que se desarrolló con aumentos de la mortalidad, con caídas de menor o mayor intensidad, dependiendo del momento histórico, de la utilización por parte de la sociedad de recursos para combatirla y de las condiciones de vida de la población a la que afectó.

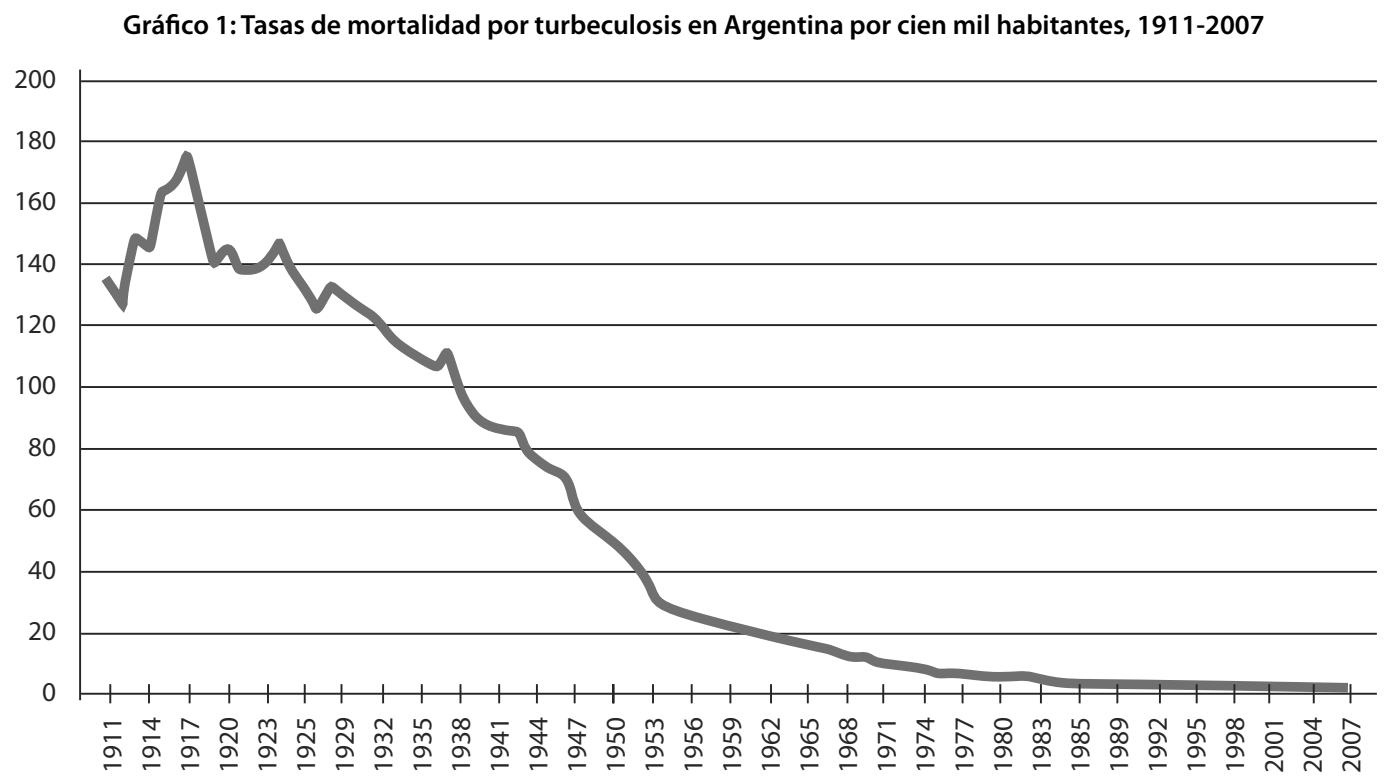

Fuente: Botinelli (1954) y estadística suministrada por el Instituto Nacional de Enfermedades Respiratorias Dr. Emilio Coni

\section{Mortalidad por tuberculosis en Argentina: la etapa pre-quimioterápica}

La mortalidad por tuberculosis tiene una fase ascendente entre 1911 y 1918 y un posterior período descendente desde 1918 a 2007. Sin embargo, ese descenso tuvo diferentes ritmos: uno más lento en la etapa anterior a los medicamentos y una aceleración a partir de 1947, momento en que comenzaron a aplicarse las primeras quimioterapias.

En la primera etapa, de ascenso de la tasa de mortalidad, ésta creció un 16\% y alcanzó el pico más alto en 1918 con 175 muertes por cada cien mil habitantes. En 1913 se publicó el Anuario Demográfico de 1911, con un detallado estudio de la mortalidad en el país realizado por el presidente del Departamento Nacional de Higiene. Citando el mencionado anuario, (Veronelli, Veronelli, 2004, p.396) los autores señalan que de las 125.727 defunciones registradas en 1911, 9.985 correspondían a muertes por tuberculosis, lo que representaba aproximadamente el 7,98\% del total. En 1914 esa proporción había pasado al 9,72\% (Veronelli, Veronelli, 2004). Entre 1916 y 1917 se aprecia una estabilización de la mortalidad y un aumento hacia 1918, año en que la pandemia de gripe española impactó en la población argentina, llevando ese porcentaje al $12,4 \%$.

Este crecimiento estaría dado por la confluencia del número de enfermos por tuberculosis y la pandemia de gripe que azotó a todo el mundo en ese año y que tuvo su impacto en 
Argentina (Carbonetti, 2011). Los médicos que estudiaban el fenómeno de aumento de la mortalidad por tuberculosis lo atribuían a dos causas fundamentales: la crisis que se habría generado como consecuencia del desarrollo de la Primera Guerra Mundial y la epidemia de gripe que se desató en 1918 (Wolaj, 1939, p.872).

A partir de 1918 comienza, como dijimos anteriormente, un descenso casi ininterrumpido hasta llegar a las tasas actuales de mortalidad por tuberculosis en el país.

El período comprendido entre 1918 y 1930 está caracterizado por una caída irregular de la mortalidad. En todo este subperíodo la tuberculosis desciende un $26 \%$. En esta etapa se construye y entra en funcionamiento una gran parte del aparato sanitario para combatir la enfermedad, especialmente sanatorios y dispensarios. La Dirección Nacional de Higiene hipotetizaba que la entrada en funcionamiento del aparato sanitario fue uno de los factores que determinaron que aquella siguiera descendiendo, incluso durante los años de la crisis mundial que comenzara en 1929 (Sussini, Paso, Zauchinger, 1935, p.22).

Entre 1931 y 1946, y a diferencia del período anterior, se observa un descenso constante del número de fallecimientos hasta alcanzar una tasa de 72,6 por cada cien mil habitantes, es decir al término de 15 años, la tasa de mortalidad por tuberculosis disminuyó un 42,4\%. ¿Cuáles serían los factores que habrían determinado dicha disminución?

Esta cuestión nos remite a la perspectiva revisionista que Simon Zsreger (1997b) desarrolló sobre la problemática de la transición demográfica y especialmente sobre la caída de la mortalidad. Apelando a un análisis de tipo político e histórico para Inglaterra, el autor observa que no bastaba con el desarrollo económico para la reducción de la mortalidad, sino que era necesario un acuerdo político que implicara el desarrollo de instituciones que permitieran, mediante la atención médica y social, la reducción de casos. Específicamente se concentra en mostrar la complejidad histórica de las relaciones entre crecimiento económico y políticas de salud y condiciones de salud, conjeturando que los modelos de las ciencias sociales más influyentes de post-guerra, como la teoría de la transición demográfica y la tesis de McKeown (1978) de las mejoras nutricionales de conducción al descenso de la mortalidad, fueron construidos teleológicamente como guías de acción positiva en el presente y el futuro. Zsreter se centra en los resultados de las políticas del movimiento de salud pública a partir de la evidencia británica desde fines del siglo XVIII hasta mediados del siglo XIX, momento en el que ésta se constituía en la primera nación industrial del mundo. Este caso permite, al autor, apreciar que la escala y la naturaleza de la disrupción política es la dimensión más importante que influye en la salud. En este proceso es críticamente determinante la capacidad de la sociedad, del Estado, de los ciudadanos y de sus asociaciones y unidades administrativas para lograr estrategias exitosas que administren las disrupciones del cambio económico.

La Oficina Sanitaria Panamericana detallaba, en una publicación de 1936, cómo se fueron constituyendo las diferentes instituciones que se encargaron del combate a la tuberculosis, que se situaban en Buenos Aires y Córdoba, ciudades hacia donde se dirigía el mayor flujo de inmigración de enfermos de las demás provincias del país:

La lucha antituberculosa en la ciudad de Buenos Aires puede decirse que comenzó con la fundación en 1901 de la Liga Argentina contra la Tuberculosis por Coni. En 1902 se dictaron las primeras ordenanzas relativas a la declaración obligatoria de los casos, desinfección de habitaciones ocupadas por éstos, prohibición de ciertos oficios a los 
enfermos, prohibición de escupir en el suelo, análisis gratuitos del esputo, etc. En 1904 se construyó el primer establecimiento destinado clínicamente a tuberculosos, es decir, el Hospital Tornú, con capacidad para 96 enfermos, que se ha ido ampliando hasta que hoy día contiene 700 camas para ambos sexos. El Hospital Muñiz, sin embargo, ya tenía servicios de tisiología y los tiene aún, muy bien organizados. En 1920 se crearon 5 dispensarios antituberculosos y en 1925 dos más. En 1924 se aprobó la construcción de la maternidad para tuberculosas en el Hospital Tornú y después se fundó el Preventorio Roca para niños hasta de 8 años. La Colonia Marítima de Necochea vino después, permitiendo recibir a 500 niños más. En la lucha también participan el Departamento Nacional de Higiene, los dispensarios nacionales, las ligas Argentina Israelita contra el mal, diversas mutualidades, y la Sociedad de Beneficencia. (OSP, 1936, p.876).

En el caso de Córdoba, en especial luego de 1920, también se crearon una gran cantidad de establecimientos, en número de camas, de todo tipo, que albergaron enfermos o sólo los atendieron. Según Huber (2000, p.23), al comenzar a funcionar el complejo Sanatorio, dirigido y ampliado por el Estado Nacional en 1915, contaba con cuatrocientas camas. Hacia 1925, ese número se había cuadruplicado, llevándolo a mil quinientas camas. Dichos enfermos eran atendidos por ochocientos empleados. A eso se sumaba una gran cantidad de sanatorios privados, como el Sanatorio Mieres, la Clínica Berna, el Hogar Japonés, el Sanatorio de la Marina, el Sanatorio Laennec y el Centro Universitario.

A esto se agregaron dos sanatorios más como el Sanatorio de Nuestra Señora de la Misericordia y el Hospital Tránsito Cáceres de Allende, además de un pabellón dedicado a tuberculosos del Hospital Rawson. Todos estos establecimientos fueron creados hacia el año 1922. Hacia mediados de la década de 1930, estos establecimientos sumaban aproximadamente quinientas camas (Carbonetti, 2011, p.201-231). A estos se sumaban aquellos que atendían a pacientes ambulatorios, como el dispensario de la Sociedad Tránsito Cáceres de Allende (1918), el Dispensario antituberculoso, dependiente del Hospital Rawson (1926), y el Dispensario Central Antituberculoso, dependiente del gobierno de la provincia de Córdoba (1931) (Carbonetti, 2011, p.203).

Si los médicos citados anteriormente acertaban al diagnóstico de la situación y el factor que estaría influyendo sobre la baja en la mortalidad (debemos recordar que no sólo actuaban los hospitales donde había internación sino también los dispensarios en los que se les suministraba comida a los enfermos y distintos tipos de atención, así como la detección temprana de la enfermedad), se puede considerar, a modo de hipótesis, un triple proceso que contribuyó a este comportamiento. En primer lugar, un aumento del aparato sanitario en relación a 1918 a fin de que aquellos que enfermaran tuvieran atención médica y social en los sanatorios y dispensarios. En segundo lugar, el impacto de la crisis en Argentina fue menor que en los países centrales y las condiciones económicas que desarrolló el país implicó una dieta rica en proteínas, basada en la carne, que es posible haya tenido algún tipo de influencia en la disminución del desarrollo de la enfermedad. En tercer lugar, el proceso de 'tuberculinización', por el cual, una vez ingresada la enfermedad en segunda y tercera generación, los sujetos de una población adquieren anticuerpos en contra de la enfermedad evitando el desarrollo de la misma. 


\section{Mortalidad por tuberculosis en Argentina: el advenimiento de la quimioterapia}

El advenimiento de las quimioterapias en el tratamiento de la tuberculosis no generó un comportamiento en términos de la mortalidad uniforme. En esta etapa es posible apreciar tres períodos: 1947-1958, 1959-1984 y 1985-2007. A partir de 1947 y hasta 1958, la tasa de mortalidad cae un 70\%. Este fenómeno está marcado por la implementación de las primeras quimioterapias contra la tuberculosis: la estreptomicina, que aparece a mediados de la década del cuarenta, al igual que el ácido paraaminosalicílico (PAS). Unos años más tarde se descubre la isoniacida, considerado el más eficaz de los medicamentos antituberculosos que ofrecía además otras ventajas: su bajo costo, los enfermos la tomaban con facilidad y ocasionaba muy pocas complicaciones cuando se la administraba en las dosis necesarias para que el tratamiento fuera eficaz (OMS, 1958).

En este período se producen dos etapas bianuales (1947-1948 y 1953-1954) donde el decrecimiento es muy pronunciado. Este es el lapso de tiempo en el que la mortalidad por tuberculosis cae a mayor ritmo: desde 1918 hasta 1946, es decir durante 28 años, la mortalidad por tuberculosis cayó en un 45,9\%, mientras que entre 1947 y 1958 lo hace en un 66,8\%. Este fenómeno objeta en alguna medida la tesis postulada por McKeown (1978, p.199-200), quien considera que "no debe causar contrariedad alguna la conclusión de que las medidas médicas de inmunización y tratamiento fueron ineficaces; también innecesarias" pues el autor consideraba "que la salud del hombre está determinada esencialmente por su comportamiento, su alimentación y la naturaleza del mundo que la rodea y está influida sólo marginalmente por el cuidado médico personal".

La aceleración del decrecimiento de la mortalidad por tuberculosis, conjuntamente con la aparición de quimioterapias efectivas contra esta enfermedad, permite afirmar que si bien se venía produciendo una baja importante de la mortalidad, los medicamentos contribuyeron a una pronunciada caída que se traduce en la reducción de más de diez puntos, tanto entre 19471948 como 1953-1954. A este fenómeno debemos agregar la modernización de un sistema de lucha contra la enfermedad que también comenzó a ser efectivo. Durante este periodo en Argentina, la Secretaría de Salud Pública, conducida por Ramón Carrillo, incorporaba dos armas en el tratamiento de la tuberculosis ya mencionadas en los párrafos anteriores: la estreptomicina y la isoniacida que cambiaron radicalmente las técnicas del control de la tuberculosis, no sólo aumentando considerablemente las posibilidades de cura, sino también acortando los tratamientos y disminuyendo o suprimiendo los períodos de internación. Se puso énfasis en la asistencia gratuita al enfermo, por medio de la racionalización técnica de los servicios, y la organización de la asistencia a crónicos (entre ellos los enfermos con tuberculosis). Los dispensarios establecían el diagnostico, así como el tratamiento ambulatorio, y se constituían centros de vigilancia de contactos, mientras que los sanatorios y los hospitales tenían la finalidad de tratar y aislar los casos de infección avanzada (SSP, 1947).

En la década del cincuenta, estudios clínicos demostraron una mayor efectividad con el empleo combinado de PAS y la estreptomicina, a las que se agregó la isoniacida, mejorando significativamente la eficacia del tratamiento que, en ese entonces, debía ser administrado durante un período que iba entre los 18 a 24 meses.

Unos años más tarde, con la natural evolución experimentada en el campo de la investigación médica en el mundo, se hace visible la necesidad de cambios en la lucha contra 
el problema de la tuberculosis. De esta forma, por decisión del gobierno nacional y con el acuerdo del gobierno de la provincia de Santa Fe, el 2 de diciembre de 1961, se inaugura oficialmente el Centro Nacional de Lucha Antituberculosa, siendo su director el doctor Justo López Bonilla, iniciando las actividades en enero de 1962 con el objetivo de dar impulso y normatizar el control de la tuberculosis en la República Argentina (Iner, s.d.).

En consecuencia, el período que comienza en 1959 y que consideramos hasta 1984 será el de la consolidación de la terapia contra la tuberculosis. En 1960, el etambutol sustituyó al PAS y la duración del tratamiento se redujo a 18 meses; en la década del setenta, con la introducción de la rifampicina, como parte de la combinación de drogas antituberculosas, la duración del tratamiento disminuyó a la mitad (Ritacco, Leão, Palomino, 2007). Gracias a los avances en el tratamiento, este período se caracteriza por una mayor caída de la mortalidad por tuberculosis. Al finalizar el período la tasa de mortalidad por tuberculosis cayó, aproximadamente, cinco veces el valor que tenía en su inicio. Sin embargo, a pesar de cierta regularidad hacia 1970, es posible observar un crecimiento de la mortalidad por tuberculosis, asociado, probablemente, a un aumento de la mortalidad y la morbilidad de enfermedades de tipo contagioso en la Argentina (Carbonetti, Celton, 2007).

En conclusión, en las décadas del cincuenta-sesenta, la incorporación de la quimioterapia moderna y la declinación de la enfermedad, y en especial de la mortalidad, marcan una nueva era en la historia de la tuberculosis. Si bien no debe olvidarse que el descenso de la mortalidad por tuberculosis ya había comenzado muchos años antes debido a varios factores, tales como mejoras en las condiciones socioeconómicas de la población, adelantos nutricionales y ambientales, nuevas medidas en el control de la enfermedad, la inmunización natural de la población y mejoras en el acceso a la atención.

El último período analizado ${ }^{1}$ se caracteriza por la aparición del VIH/Sida, momento en el cual la tuberculosis se constituye en la primera enfermedad marcadora - además prolonga la duración de los tratamientos, genera formas más graves y reduce las posibilidades de cura. En esta etapa, la tendencia de la mortalidad por tuberculosis comenzó a ser irregular nuevamente. Se inicia con un mayor decrecimiento del que se venía dando hasta el momento en la década de 1980, pero, en la década de 1990, se observa un nuevo aumento, posiblemente influenciado por la retirada del Estado frente a las reformas neoliberales y el impacto del VIH que empezó a incidir en la curva y, como sucedió a nivel mundial, en este período se observa un recrudecimiento de la morbimortalidad.

La tendencia de la mortalidad por tuberculosis, entre 1985 y 2000, ha descendido con ciertas variaciones a lo largo del período (Gráfico 2), tanto en relación a las muertes por tuberculosis (tuberculosis con o sin VIH) y las muertes por tuberculosis (tuberculosis sin VIH). De este modo, se puede observar la separación de las tendencias a partir de 1989. Teniendo en cuenta la mortalidad por tuberculosis (con o sin VIH), el descenso en todo el período es de $3,1 \%$ anual promedio, pero si se considera sólo la mortalidad por tuberculosis (sin VIH), la tendencia al descenso aumenta a $4,2 \%$.

Por otra parte, la tendencia al descenso de la mortalidad registró su valor más importante entre 1980 y 1985, donde todavía no había muertes registradas por la combinación de ambas causas (tuberculosis y VIH): 9,0\% anual promedio (Iner, 2002). De esta forma, el número de muertes por esta causa registraba un descenso del 45\% entre 1980 y 2001, siendo este 
ritmo, como puede observarse, mayor durante el primer decenio (1980-1990) que durante el segundo decenio (1991-2001).

En 2001 se registraron 936 muertes por tuberculosis de todas las formas, lo que representó una tasa de 2,5 por cien mil habitantes. De estas muertes, 135 estuvieron asociadas a Sida. La tendencia de la mortalidad por tuberculosis fue al descenso desde 1980 y el número de muertes por tuberculosis registrado en 2001 fue inferior al de 2000 y el más bajo de todo el período (Iner, 2003). Sin embargo en el año 2002, por primera vez en muchos años, y luego de un largo período de descenso, se observa un aumento de la tasa de mortalidad que pudo deberse a la crisis social, económica y política por la que atravesó el país y que tuvo un impacto en varios indicadores de salud. Incluso algunos estudios señalan que esta situación de crisis ha afectado de manera desigual a los diferentes estratos sociales expresados en los indicadores de mortalidad general (Carbonetti, Celton, 2007).

En nuestro país, la variación de la mortalidad a lo largo de la historia se ha debido a diversas causas que abarcan desde la declinación natural de la enfermedad hasta los cambios políticos, sociales y económicos, junto con los avances científicos. Esta combinación de factores es lo que subyace detrás de las fluctuaciones, más o menos pronunciadas, de la curva en casi cien años. Ahora bien, como sucede a nivel mundial, la distribución de la mortalidad por tuberculosis en nuestro país no ha sido y no es uniforme. La tasa de mortalidad por tuberculosis en la Argentina oculta diferentes situaciones, según el área geográfica. En consecuencia, a continuación se presenta el comportamiento de la mortalidad por tuberculosis en las distintas regiones argentinas, tomando algunos años claves en la primera mitad del siglo XX y los años censales en la segunda mitad de esa centuria.

Gráfico 2: Tasas de mortalidad por turbeculosis total y sin VIH en Argentina, 1985-2007

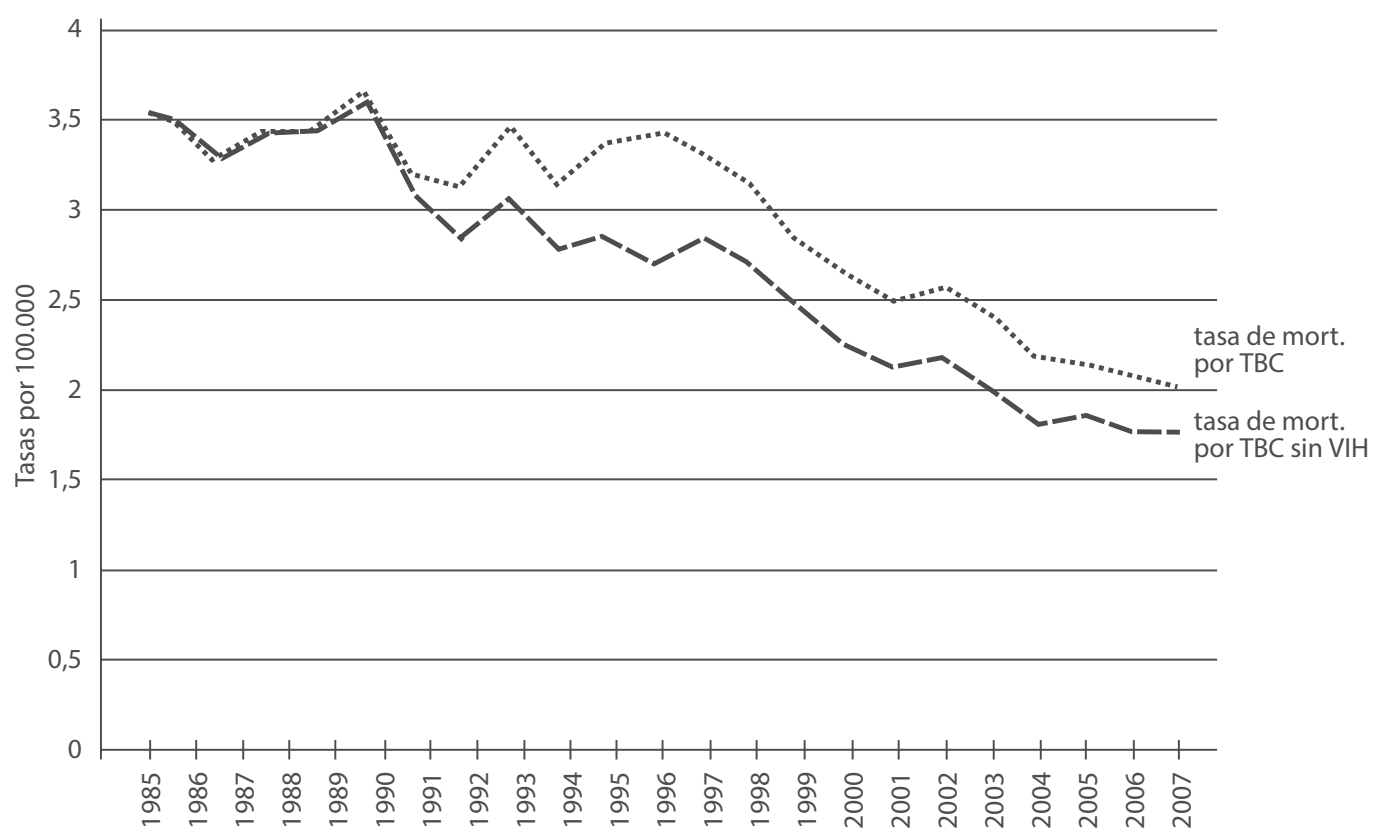

Fuente: Estadística suministrada por el Instituto Nacional de Enfermedades Respiratorias Dr. Emilio Coni 


\section{La mortalidad por tuberculosis en las regiones argentinas}

La mortalidad por tuberculosis en Argentina presenta un panorama muy heterogéneo en términos territoriales a lo largo del siglo XX. Por ello hemos elaborado regiones tomando en cuenta factores como desarrollo económico y social, crecimiento demográfico, criterios geográficos y de obtención de datos. Así la región central está conformada por las provincias de Buenos Aires, Córdoba, Santa Fe y la Ciudad Autónoma de Buenos Aires; la Mesopotamia, por Entre Ríos y Corrientes; Cuyo, por Mendoza, San Juan y San Luis; Noroeste, por La Rioja, Catamarca, Jujuy, Salta, Tucumán y Santiago del Estero; Noreste, por Chaco Formosa y Misiones²; y Patagonia, por La Pampa, Río Negro, Chubut, Santa Cruz y Tierra del Fuego.

El Gráfico 3 refleja lo sucedido con la mortalidad por tuberculosis en las regiones antes mencionadas y que permitiría formular la hipótesis de una acompañamiento de esta dolencia en relación a la transición epidemiológica caracterizada, justamente, por una fuerte heterogeneidad (Carbonetti, Celton, 2007, p.381). Según datos analizados por estos dos autores, "las condiciones económicas y sociales así como las políticas de salud generaron un contexto epidemiológico para que, en el momento de inicio de la transición, las distintas regiones que integran la Argentina tuvieran condiciones de salud distintas. Eso generó un retraso que se ha podido observar en los distintos indicadores". Este fenómeno de retraso se puede apreciar en especial en las regiones del Noroeste y el Noreste que partieron de una esperanza de vida menor a las de las demás regiones. Esta variable podría indicarnos, desde el punto de vista de la mortalidad por tuberculosis, subregistros. No obstante este fenómeno estadístico es difícil de ser demostrado.

Gráfico 3: Tasas de mortalidad por turbeculosis según regiones de la Argentina, por cien mil habitantes, 1914-2007

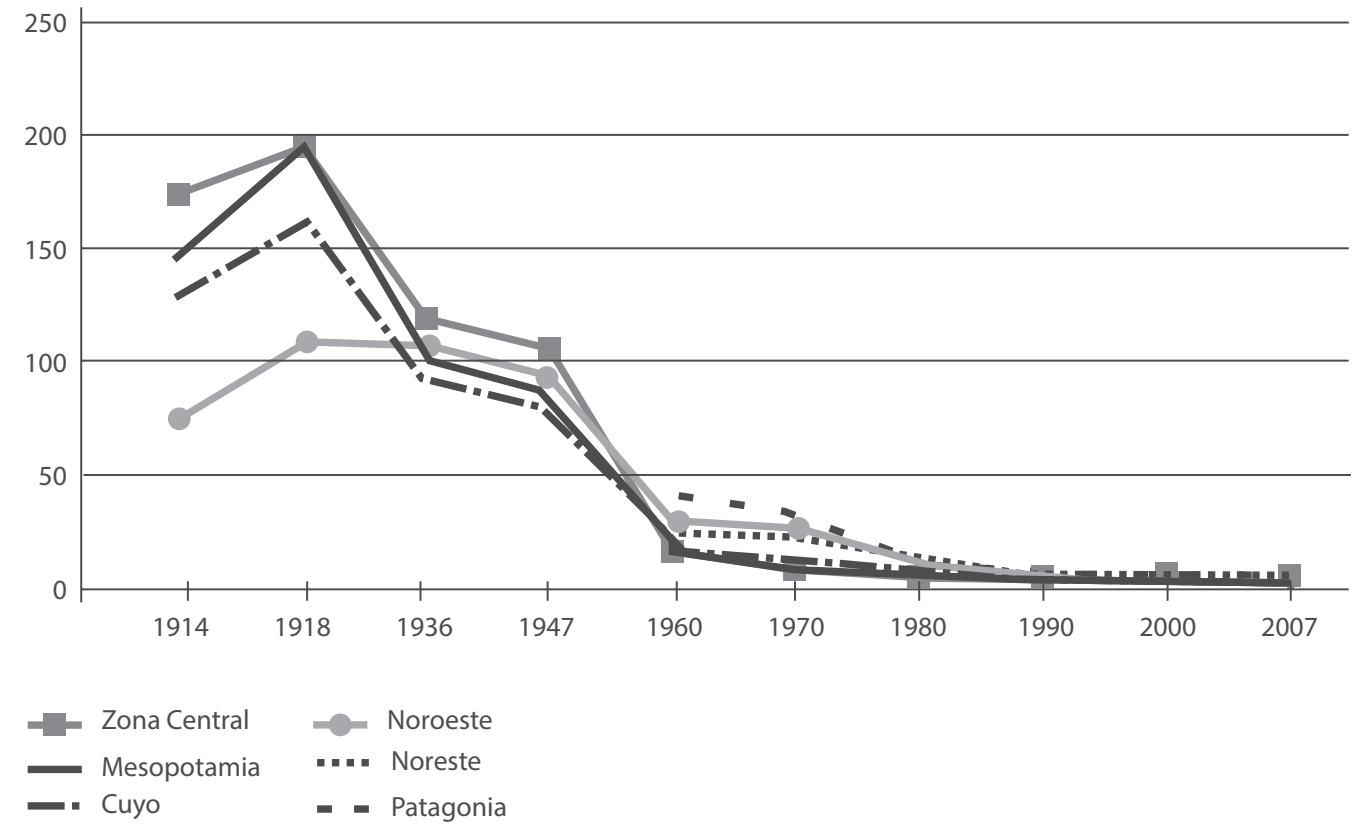

Fuente: Elaboración propia sobre la base de Sayago $(1921,1943)$ 
Hacia el comienzo del período de estudio, la mortalidad por tuberculosis tenía una mayor predominancia especialmente en la región Central, la Mesopotamia y Cuyo, aunque estas últimas con niveles menores. La región del Noroeste refleja un nivel muy por debajo de las anteriores. Luego de 1918, momento en que se produce la tasa más alta, las tres regiones que tenían niveles elevados (Central, Cuyo y Mesopotamia) tienden a decrecer su mortalidad mientras que la región del Noroeste no se modifica generando un amesetamiento en el comportamiento de la mortalidad.

Entre 1936 y 1947, momento en el que es posible se haya ajustado el sistema de registro de muertes, los niveles de mortalidad por tuberculosis del Noroeste sólo eran superados por la región Central, si bien esta región era la de mayor desarrollo económico y social, también tuvo algunas características que la hacían la zona de mayor mortalidad por tuberculosis. Allí se encontraba el 83\% de las 9.475 camas destinadas al tratamiento de la tuberculosis (Sussini, Paso, Zauchinger, 1935), lo que hace suponer cierta inmigración de enfermos hacia esas provincias y que en muchos casos encontraban el fin de la vida en esta región. Es posible que esto haya generado que, a pesar de la baja importante que tuvo entre las fechas antes mencionadas, continuara siendo la región con mayores tasas de mortalidad por tuberculosis.

El período en el que generaría cambios importantes en los niveles de mortalidad por tuberculosis, tanto en Argentina como en sus distintas regiones, es el de 1947-1960, momento en el cual comenzaron a aplicarse las primeras quimioterapias y que, como veíamos en párrafos anteriores, produjo en el país una baja substancial. En consecuencia, se observa que fueron las regiones con menores niveles de desarrollo, como el Noroeste, Noreste y Patagonia, aquellas que sufrieron una mortalidad más alta en momentos en que se desarrollaba en toda su plenitud el andamiaje institucional y se incorporaban los medicamentos más efectivos.

Este fenómeno puede deberse a que aquellas regiones que habían sufrido el mayor impacto de la tuberculosis en la era previa al surgimiento de los medicamentos tenían ya consolidado un aparato sanitario que les permitió acelerar la baja de la mortalidad, mientras que las regiones con provincias más pobres y con escaso andamiaje sanitario fueron las que conservaron tasas de mortalidad más elevadas. En esta etapa, la región Central acelera el ritmo de decrecimiento de la mortalidad y hacia el final del período ya se encontraba con tasas por debajo de las demás regiones.

A partir de 1960 y hasta 1990 se produce un cambio. Las tasas tienden a crecer y estabilizarse en las regiones de Cuyo y Mesopotamia, ubicándose en niveles similares a las regiones más atrasadas como Noreste, Noroeste y Patagonia, mientras que en la región Central las tasas de mortalidad tienden a decrecer más rápidamente. Este fenómeno se aprecia en el Gráfico 4 que expresa las tasas en escala logarítmica.

Una primera hipótesis de este comportamiento estaría dada porque en la región Central habría una mayor y mejor distribución de los medicamentos, mayor medicalización y las condiciones económico-sociales serían más óptimas. Es posible también que se haya dado en la población una creciente adquisición de inmunidades al bacilo y una disminución de las migraciones de enfermos a la región Central donde se encontraba gran parte de las instituciones de tratamiento a la enfermedad en la etapa previa a la aparición de los medicamentos. 


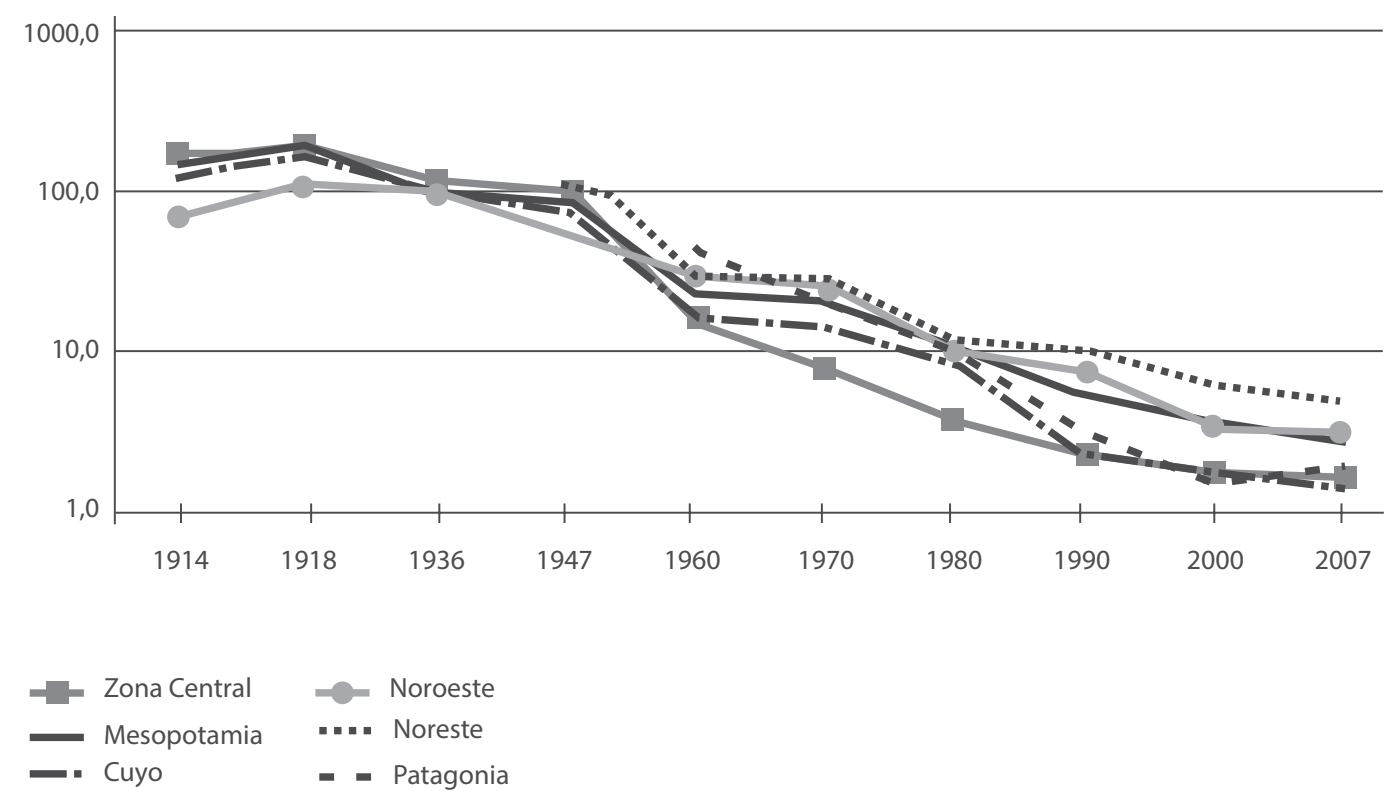

Fuente: Elaboración propia sobre la base de Sayago $(1921,1943)$

A partir de la década del noventa, las regiones de Cuyo y Patagonia tienden a ubicarse en niveles similares a los de la región Central, mientras que Mesopotamia, Noroeste y Noreste, no obstante la baja, se encuentran en un nivel más alto.

El desarrollo de políticas destinadas a combatir la enfermedad y las condiciones económicosociales influyen en el comportamiento de las tasas de mortalidad de las diferentes regiones en la etapa posterior a 1990. Son las regiones con menor nivel de desarrollo económico las que sufren tasas más altas de mortalidad: Noroeste, Noreste y Mesopotamia (influida por Corrientes y Misiones) mientras que Patagonia, Cuyo y región Central, donde más se aplicaron políticas de salud y, además de mayor desarrollo económico-social, las que lograron rebajar sus tasas de mortalidad por tuberculosis a niveles mínimos.

\section{Consideraciones finales}

A lo largo del siglo XX, la curva descripta en este artículo, tiene una continua tendencia decreciente luego de 1918 con fluctuaciones y descensos más o menos abruptos. En la aceleración de los cambios en la mortalidad por tuberculosis, los antibióticos desempeñaron un rol fundamental, no sólo en el descenso marcado a partir de la segunda mitad del siglo $\mathrm{XX}$, sino también en los avances por obtener un mayor control de la enfermedad. De esta forma, la segunda mitad de la década de 1940 y toda la década de 1950 fueron decisivas en la aceleración de la caída de la mortalidad por tuberculosis en Argentina.

Desde esta perspectiva, la historia de la mortalidad por tuberculosis se divide en dos grandes etapas: antes y después del tratamiento. Como pudimos observar antes de las quimioterapias, no sólo se calculaba que el 50\% de los enfermos morirían por esta enfermedad - por lo cual 
las tasas eran mucho más elevadas - sino que el riesgo de infección, el de enfermar y el de morir por tuberculosis en una comunidad estaban fuertemente relacionados.

En ese sentido, el análisis realizado en este artículo demuestra ciertos aspectos hasta ahora no trabajados por la historia y la sociología de la salud. Por un lado, el decrecimiento que se dio a partir de 1918, sin que existiera un tratamiento efectivo, permite entender cómo el Estado y la sociedad generaron recursos para combatirla mediante la conformación de instituciones que, si bien es posible no hayan alcanzado a internar y tratar a todos los que enfermaban, contribuyeron a disminuir la tasa de mortalidad ya que si bien no era posible la cura permitieron un retraso en las muertes mediante la provisión de diversos tratamientos, alimentación, resguardo y detección temprana de la enfermedad. A esto se debe agregar cambios en las condiciones habitacionales, de alimentación y ambientales y al mismo tiempo un proceso por el cual las distintas generaciones fueron adquiriendo una inmunidad natural a la enfermedad.

Es necesario añadir también a este fenómeno las desigualdades en términos epidemiológicos que se dieron a lo largo del siglo XX en las distintas regiones del país y que se reflejaron en la curva de la mortalidad por tuberculosis en cada una de ellas. Es posible considerar, a partir de estudios relacionados a la transición epidemiológica en las regiones de mayor subdesarrollo en el país, un subregistro, en especial en el Noroeste, una vez que comenzó a asentarse el sistema de registros y, cuando empezaron a actuar los tratamientos efectivos, la tasa de mortalidad en estas regiones fue superior, conservándose así hasta el fina del período de estudio.

A partir de la introducción del tratamiento, la magnitud y tendencia de la tasa de mortalidad se vio afectada por la eficacia de las drogas, de manera que se produjo un cambio en la relación entre adquirir la infección y desarrollar la enfermedad y morir por la misma. Este cambio se basa, principalmente, en que la relación dejó ya de depender fundamentalmente del curso natural de la enfermedad y de su proceso patológico propio para ser un reflejo de la eficacia del tratamiento implementado en gran escala en la comunidad, es decir, de la efectividad del mismo (Iner, 1988).

De esta forma, una vez demostrada la eficacia del tratamiento, las muertes cuando se producen quedan asociadas al retardo en el diagnóstico o a la edad avanzada del paciente y, fundamentalmente, a las medidas de aplicación del tratamiento y a la organización de la atención en particular y de los programas en general. Otro de los cambios fundamentales es que dado que no debieran registrarse muertes por tuberculosis, la magnitud de la tasa de mortalidad y, fundamentalmente, su tendencia dejan de constituir un índice epidemiológico para pasar a ser una expresión de la efectividad del programa de tratamiento por lo que en la actualidad puede ser utilizada para la evaluación del impacto del mismo (Iner, 1988).

Siendo una enfermedad curable y con tratamientos efectivos disponibles, actualmente el análisis de la mortalidad por tuberculosis está relacionado con la accesibilidad y oportunidad del tratamiento de la enfermedad. De todas formas, cabe mencionar que la aparición del VIH/ Sida y las resistencias a los tratamientos, como así también la problemática de la adherencia a los tratamientos, plantean nuevos desafíos por sus efectos sobre la efectividad de los tratamientos y los riesgos de muerte.

Por otra parte, el desarrollo de las quimioterapias permitió un decrecimiento de la mortalidad por tuberculosis que lo llevó a sus niveles mínimos hacia la década de 1980, antes 
de la aparición del Sida en el horizonte epidemiológico. Este comportamiento general no fue uniforme, sino que dependió de las características socioeconómicas de las diferentes regiones, de los momentos históricos en que la tuberculosis la afectó y del grado de preparación y desarrollo de los instrumentos técnicos e institucionales para el combate a la enfermedad.

\title{
NOTAS
}

\begin{abstract}
${ }^{1}$ La información de mortalidad que genera el país en este último período se concibe a través de los certificados de defunción que se producen en todo el país y que son procesados anualmente por las oficinas del Registro Civil y, en segundo término, por las Direcciones Provinciales de Estadística. La consolidación y procesamiento final se realiza en la Dirección Nacional de Estadísticas de Salud, donde se redistribuyen las muertes registradas según su lugar de residencia habitual (Iner, 2003).

${ }^{2}$ Si bien en términos geográficos la provincia de Misiones fue tradicionalmente incorporada a la región Mesopotámica, por características de su historia social, política y económica, así como por la obtención de datos, se la ha incluido a la región Noreste.
\end{abstract}

\section{REFERENCIAS}

ARAOZ, Ricardo.

Consideraciones sobre las estadísticas demográficas de la provincia de Salta. Trabajo presentado en 1. Congreso Nacional de Medicina, 1916. Buenos Aires. 1916.

\section{ARGENTNA.}

Ministerio de Salud. Instituto Nacional de Enfermedades Respiratorias Dr. Emilio Coni. Normas técnicas del Programa Nacional de Control de la Tuberculosis. Buenos Aires: Instituto Nacional de Enfermedades Respiratorias Dr. Emilio Coni. 2006.

ARMUS, Diego.

La ciudad impura: salud, tuberculosis y cultura en Buenos Aires, 1870-1950. Buenos Aires: Edhasa. 2007.

BELDARRAÍN CHAPLE, Enrique. Apuntes para la historia de la lucha antituberculosa en Cuba. Revista Cubana de Salud Pública, La Habana, v.1, n.2, p.97-105. 1998.

BERTOLLI FILHO, Claudio.

História social da tuberculose e do tuberculoso: 1900-1950. Rio de Janeiro: Editora Fiocruz. 2001.

BLINN REBER, Vera.

Blood, coughs, and fever: tuberculosis and the working class of Buenos Aires, Argentina, 1885-1915. Social History of medicine, Oxford, v.12, p.73-100. 1999.

BOTINELLI, Pedro.

Estadística de mortalidad por tuberculosis. Buenos Aires: Ministerio de Asistencia Social y Salud Pública/Dirección Nacional de Investigaciones Demológicas. 1954.

CARBONETTI, Adrián.

La ciudad de la peste blanca: historia epidemiológica, política y cultural de la tuberculosis en la ciudad de Córdoba, Argentina, 1895-1947. México: Fondo Editorial de la Benemérita Universidad Autónoma de Puebla. 2011.

CARBONETTI, Adrián.

Enfermedad y sociedad: la tuberculosis en la ciudad de Córdoba 1906 y 1947. Córdoba: Editorial de la Municipalidad de Córdoba. 1998.

CARBONETTI, Adrián; CELTON, Dora. La transición epidemiológica. In: Torrado, S. (Comp.). Población y bienestar en Argentina del primer al segundo centenari: una historia social del siglo XX. Buenos Aires: Edhasa. 2007.

FARMER, Paul.

Infections and inequalities: the modern plagues. Los Angeles: University of California Press. 1999.

GONZÁLEZ OCHOA, Edilberto et al.

Mortalidad por tuberculosis en Cuba, 1902-1997. Revista Cubana de Medicina Tropical, La Habana, v.55, n.1, p.5-13. 2003.

HUBER, Norberto.

El Santa María de ayer: la estación climatérica y el Hospital Colonia. Córdoba: Copiar. 2000.

IDROVO, A.

Raíces históricas sociales y epidemiológicas de la tuberculosis en Bogotá, Colombia. Revista Biomédica, Bogotá, v.24, n.4, p.356-365. 2004.

INER.

Instituto Nacional de Enfermedades Respiratorias Dr. Emilio Coni. Situación de la tuberculosis. República Argentina. Santa Fe: Instituto Nacional de Enfermedades Respiratorias Dr. Emilio Coni. 2009.

INER.

Instituto Nacional de Enfermedades Respiratorias Dr. Emilio Coni. Mortalidad por tuberculosis en 
la República Argentina 2001. Santa Fe: Instituto Nacional de Enfermedades Respiratorias Dr. Emilio Coni. 2003.

INER.

Instituto Nacional de Enfermedades Respiratorias Dr. Emilio Coni. Mortalidad por tuberculosis en la República Argentina 1980-2000. Santa Fe: Instituto Nacional de Enfermedades Respiratorias Dr. Emilio Coni. 2002.

INER.

Instituto Nacional de Enfermedades Respiratorias Dr. Emilio Coni. Tuberculosis en la República Argentina: morbilidad y mortalidad 1973-1986. Santa Fe: Instituto Nacional de Enfermedades Respiratorias Dr. Emilio Coni. 1988.

INER.

Intituto Nacional de Enfermedades Respiratorias Dr. Emilio Coni. [Información institucional]. Disponible en: http://www.anlis.gov.ar/inst/ INER/historia.html. Acceso en: 31 jul. 2010. s.d.

LEÃO, Sylvia Cardoso; PORTAELS, Françoise. History. In: Palomino, Juan Carlos; Leão, Sylvia Cardoso; Ritacco, Viviana. (Ed.). Tuberculosis 2007: from basic science to patient care. S.l.: s.n. 2007. Disponible en: http://www. freebooks4doctors.com/pdf/tuberculosis2007. pdf. Accesso en: 03 abr. 2013. 2007.

MCKEOWN, Thomas.

El crecimiento moderno de la población. Barcelona: Bosch. 1978.

\section{MÉDICOS SIN FRONTERAS.}

Sin aliento: el tratamiento de la tuberculosis en el siglo XXI: informe basado en la investigación realizada por May Moran para Médicos

Sin Fronteras: Campaña para el Acceso a Medicamentos Esenciales. Ginebra: Médicos Sin Fronteras. 2006.

OMS.

Organización Mundial de la Salud. Tuberculosis. In: OMS. Los diez primeros años de la organización Mundial de la Salud. Ginebra: OMS. p.188-197. 1958. Disponible en: http:// whqlibdoc.who.int/publications/14604_(13).pdf. Accesso en: 31 jul. 2010. 1958.

OPS.

Organización Panamericana de la Salud. Situación epidemiológica de la tuberculosis. Washington: Organización Panamericana de la Salud. 2004.

OSP.

Oficina Sanitaria Panamericana. Crónicas. Boletín de la Oficina Sanitaria Panamericana, v.15, n.9, p.873- 907. 1936. Disponible en: http://hist. library.paho.org/Spanish/BOL/v15n9p873.pdf. Acceso en: 15 jun. 2011. 1936.

OSP.

Oficina Sanitaria Panamericana. Tuberculosis. Boletín de la Oficina Sanitaria Panamericana, v.13, n.12, p.1124-1157. 1934. Disponible en: http:// hist.library.paho.org/Spanish/BOL/v13n12p1124. pdf. Acceso en: 15 jun. 2011. 1934.

PÔRTO, Ângela.

A vida inteira que podia ter sido e que não foi: trajetória de um poeta tísico. História, Ciências, Saúde-Manguinhos, Rio de Janeiro, v.6, n.3 , p.523-550. 2000.

RECALDE, Héctor.

La salud de los trabajadores en Buenos Aires (18701910): a través de las fuentes médicas. Buenos Aires: Grupo Editor Universitario. 1997.

RITACCO, Viviana.; LEÃO, Sylvia Cardoso.; PALOMINO, Juan Carlos

New developments and perspectives. In: Palomino, Juan Carlos; Leão, Sylvia Cardoso; Ritacco, Viviana. (Ed.). Tuberculosis 2007: from basic science to patient care. S.l.: s.n. 2007. Disponible en: http://www.freebooks4doctors. com/pdf/tuberculosis2007.pdf. Acceso en: 03 abr. 2013. 2007.

SAYAGO, Gumersindo.

Demografía de la tuberculosis en la provincia de Córdoba. Temas de Tisiología, Córdoba, t.1, n.3, p.945-956, 1943.

SAYAGO, Gumersindo.

La tuberculosis en la provincia de Córdoba.

Córdoba: s.n. 1921.

SSP.

Secretaria de Salud Pública de la Nación. Plan analítico de salud pública. Buenos Aires: Secretaria de Salud Pública de la Nación. 1947.

SUSSINI, Miguel; PASO, Juan Roberto.; ZAUCHINGER, Adela.

La tuberculosis en la Argentina, evolución de la mortalidad. Anales del Departamento Nacional de Higiene, Buenos Aires, n.36, p.5-95. 1935.

SZRETER, Simon.

Health and wealth: economic growth, disruption, deprivation, disease, and death. Population and Development Review, Malden, v.23, n.4, p.693-728. 1997a.

SZRETER, Simon.

Health and wealth: studies in history and policy. Rochester: University of Rochester Press. 1997b.

VERONELLI, Juan Carlos; VERONELLI Correch, Magalí.

Los orígenes institucionales de la Salud Pública en la Argentina. t.2. S.l.: Organización Panamericana de la Salud. 2004.

WOLAJ, Isaac.

Características de la infección tuberculosa en nuestro país y su importancia para la lucha antituberculosa. In: Congreso Nacional de Medicina, 6., 1938. Actas y trabajos... Rosario: Establecimientos Pomponio. 1939. 